Constraining decaying dark matter with Fermi LAT gamma-rays

This article has been downloaded from IOPscience. Please scroll down to see the full text article.

JCAP06(2010)027

(http://iopscience.iop.org/1475-7516/2010/06/027)

View the table of contents for this issue, or go to the journal homepage for more

Download details:

IP Address: 131.169.87.206

The article was downloaded on $28 / 06 / 2010$ at $12: 42$

Please note that terms and conditions apply. 


\title{
Constraining decaying dark matter with Fermi LAT gamma-rays
}

\section{Le Zhang, ${ }^{a}$ Christoph Weniger, ${ }^{b}$ Luca Maccione, ${ }^{b}$ Javier Redondo ${ }^{c}$ and Günter Sigl ${ }^{a}$}

\author{
${ }^{a}$ II. Institut für theoretische Physik, Universität Hamburg, \\ Luruper Chaussee 149, D-22761 Hamburg, Germany \\ ${ }^{b}$ Deutsches Elektronen-Synchrotron, \\ Notkestraße 85, D-22607 Hamburg, Germany \\ ${ }^{c}$ Max-Planck-Institut (Werner Heisenberg Institut), \\ Föhringer Ring 6, D-80805 München, Germany \\ E-mail: lezhang@mail.desy.de,christoph.weniger@desy.de, luca.maccione@desy.de, \\ redondo@mppmm.mpg.de, sigl@mail.desy.de
}

Received February 15, 2010

Revised April 20, 2010

Accepted June 1, 2010

Published June 28, 2010

\begin{abstract}
High energy electrons and positrons from decaying dark matter can produce a significant flux of gamma rays by inverse Compton off low energy photons in the interstellar radiation field. This possibility is inevitably related with the dark matter interpretation of the observed PAMELA and FERMI excesses. The aim of this paper is providing a simple and universal method to constrain dark matter models which produce electrons and positrons in their decay by using the Fermi LAT gamma-ray observations in the energy range between $0.5 \mathrm{GeV}$ and $300 \mathrm{GeV}$. We provide a set of universal response functions that, once convolved with a specific dark matter model produce the desired constraints. Our response functions contain all the astrophysical inputs such as the electron propagation in the galaxy, the dark matter profile, the gamma-ray fluxes of known origin, and the Fermi LAT data. We study the uncertainties in the determination of the response functions and apply them to place constraints on some specific dark matter decay models that can well fit the positron and electron fluxes observed by PAMELA and Fermi LAT. To this end we also take into account prompt radiation from the dark matter decay. We find that with the available data decaying dark matter cannot be excluded as source of the PAMELA positron excess.
\end{abstract}

Keywords: dark matter theory, cosmic ray experiments, gamma ray experiments 


\section{Contents}

1 Introduction 1

2 Gamma-rays from decaying dark matter 3

2.1 Inverse Compton scattering and bremsstrahlung 4

$\begin{array}{lll}2.2 & \text { Prompt radiation } & 7\end{array}$

$3 \quad e^{ \pm}$-response functions from inverse Compton scattering $\quad 8$

3.1 Definitions and optimal sky patch 8

$\begin{array}{lll}3.2 & \text { Response functions without foreground subtraction } & 10\end{array}$

3.3 Response functions with subtraction of astrophysical foregrounds 11

4 Constraints on dark matter models $\quad 14$

5 Conclusions

\section{Introduction}

The existence of dark matter in our Universe is nowadays a widely accepted fact. Dark matter constitutes the dominant fraction of matter present in the Universe. However, all the astrophysical evidences for dark matter, such as those coming from gravitational lensing, galaxy rotation curves and cosmic microwave background (CMB) anisotropies, are purely gravitational [1], and its particle nature remains unknown.

The most popular type of dark matter (DM) candidate, a weakly interacting massive particle (WIMP), can naturally reproduce the observed DM abundance due to effective selfannihilation in the early Universe. Today, this same annihilation process could produce an observable contribution to the measured cosmic-ray fluxes on Earth [2-5] and have impact on cosmological observations [6]. However, such an indirect detection of DM is also possible if DM decays at a sufficiently large rate. Indeed, there exists a number of interesting and theoretically well motivated DM models that predict the decay of DM on cosmological time scales, namely with lifetimes around and above $\tau_{\chi} \simeq \mathcal{O}\left(10^{26} \mathrm{~s}\right)$, which are typically required to be not in conflict with current observational limits [7-9]. Among these candidates is the gravitino in small $R$-parity breaking scenarios (motivated by requiring a consistent thermal history of the Universe [10]) and models of sterile neutrinos, whose long lifetime is due to tiny Yukawa couplings (see ref. [11] and references therein). Other interesting models include kinetically mixed hidden gauge bosons and gauginos $[12,13]$. Even in models where the DM candidate is stable in the first place, the consideration of higher-dimensional operators often renders the DM candidate particle unstable with cosmological lifetimes [14]. Since the indirect detection signals from decay differ in general from the ones of annihilation, a dedicated study of decaying DM signals is warranted.

On the observational side, recent results by several Cosmic Ray (CR) and $\gamma$-ray experiments have seriously challenged the standard description and interpretation of the generation of high-energy CR electrons, positrons and $\gamma$-rays, thus giving room also to DM interpretations. In particular, the PAMELA satellite reported the presence of an increase in the positron fraction $J\left(e^{+}\right) / J\left(e^{+}+e^{-}\right)$above $10 \mathrm{GeV}$ [15]. If there is no primary source of CR 
positrons in the Galaxy, and since secondary positrons originate from the interactions between CR nuclei (mainly protons and Helium) and the interstellar gas, their spectrum on Earth should be softer than the primary electron spectrum. Therefore, the positron fraction is expected to decrease with increasing energy. The observed rise at high energy (the so called positron fraction excess) leads then to postulate the existence of an additional primary source of positrons with energies ranging from 10 to at least $100 \mathrm{GeV}$. Although considering more carefully standard astrophysical sources, such as pulsars, provides a possible explanation for this excess [16-20], also dark matter interpretations are possible. In particular, DM decays might provide the required amount of primary positrons [12, 14, 21-24]. However, we should stress that the requirement of an additional primary positron source is not strictly necessary to interpret the data. The positron excess can be interpreted also as due to propagation effects, either in the standard sources or in the ISM. For example, the excess could be due to inhomogeneities of CR sources [25], or it could be a consequence of reacceleration of secondary cosmic rays in supernova remnants [26]. Also an increase of starlight and interstellar gas densities could reproduce the positron excess [27]. Lastly, also nested leaky box models can in principle provide a good fit to the positron fraction $[28,29]$.

Apart from the positron fraction, also the $e^{-}+e^{+}$spectra from the Fermi LAT measurement [30] and HESS [31] are intriguing. The Fermi LAT instrument measured a total $e^{+}+e^{-}$ spectrum at high energies from $20 \mathrm{GeV}$ to $1 \mathrm{TeV}$ on Earth significantly harder than expected according to propagation models, and with a hint of the presence of a broad bump at a few hundred $\mathrm{GeV}$, which is smoothly connected with HESS observations around the TeV (see, e.g. $[32,33]$ for a review of possible interpretations of these observations). This also provides a motivation to explain the excess using decaying dark matter which can easily produce large numbers of energetic positrons and electrons. Both these striking observations have motivated a plethora of works on both building and constraining new dark matter models.

High energy CR electrons and positrons produce $\gamma$-rays through the processes of inverse Compton scattering (ICS) off low energy photons in the interstellar radiation field (ISRF) and through bremsstrahlung emission due to the interaction with the interstellar medium (ISM). The Fermi Gamma-Ray Space Telescope is currently observing the $\gamma$-ray sky in the energy range between $30 \mathrm{MeV}$ and $300 \mathrm{GeV}$ with unprecedented angular resolution and precise energy sensitivity. Data obtained during the first year of operation of Fermi are about to be released officially, and preliminary data on the $\gamma$-ray spectrum in some regions of the sky were already made public in several Conferences. It is then timely to provide tools to exploit this detailed information in an efficient way.

At present, most attempts to construct dark matter candidates as the interpretation of the PAMELA and $\gamma$-ray data are inevitably model dependent. Thus, it is useful to develop model independent analysis tools such as response functions. These are functions of injected electron or positron energy which are obtained from comparing the $\gamma$-ray spectrum resulting on Earth after propagation (which we call signal) with the observed $\gamma$-ray spectrum (which henceforth will be called background). Indeed, this method has already been used to constrain decaying DM scenarios with the observed galactic synchrotron radiation maps [34]. Since the propagation equation is linear in the source term, each injected electron energy evolves independently. Therefore, with a finite number of numerical simulations at different injection energies we can construct a numerical $e^{ \pm}$-response function of signal-to-background. The $e^{ \pm}$-response function encodes our knowledge about the propagation of CR electrons in the Galaxy, as well as about the distribution of DM, but is independent of the particle physics model leading to the actual spectra of electrons and positrons after DM decay. Moreover, 
the $e^{ \pm}$-response functions we compute in this work are normalized to the present $\gamma$-ray data. Hence, constraints can be obtained by the convolution of the $e^{ \pm}$-response functions with a given DM decay spectrum and requiring that the result be smaller than the product of DM mass and lifetime in suitable units. If this condition is violated, the DM model predicts $\gamma$-ray fluxes (from ICS and bremsstrahlung) that are higher than the data by at least $2 \sigma$.

In addition to $\gamma$-rays produced by ICS and bremsstrahlung of pairs resulting from DM decay, the prompt radiation of $\gamma$-rays during the DM decay can be a very relevant signature of DM decays. However, since prompt radiation depends on the particle physics, we cannot include it in our $e^{ \pm}$-response functions and has to be included by hand when pursuing constraints on a specific model.

The paper is organized as follows. In section 2 , we describe our method of $e^{ \pm}$-response functions to provide constrains on any dark matter model involving electrons and positrons in its decay products. In section 3 we present the $e^{ \pm}$-response functions based on the Fermi data and discuss how they are improved by the removal of astrophysical contributions of known origin to the $\gamma$-ray signal. In section 4 we apply our $e^{ \pm}$-response functions to some specific dark matter decay scenarios and compare it to the contribution of prompt radiation to the $\gamma$-ray constraints. Finally, in section 5 we present our final discussions and summarize.

\section{Gamma-rays from decaying dark matter}

In this section we will discuss the different sources of gamma radiation resulting from dark matter decay. Generically, $\gamma$-rays are expected as a secondary product of the interactions of CRs with the galactic gas and radiation field. It is well known that CRs in the energy range we are interested in obey the following propagation equation (Ginzburg \& Syrovatskii [35])

$$
\begin{aligned}
\frac{\partial N^{e^{ \pm}}}{\partial t}-\nabla \cdot\left(D \nabla-\boldsymbol{v}_{c}\right) N^{e^{ \pm}}+\frac{\partial}{\partial p}(\dot{p} & \left.-\frac{p}{3} \boldsymbol{\nabla} \cdot \boldsymbol{v}_{c}\right) N^{e^{ \pm}}-\frac{\partial}{\partial p} p^{2} D_{p p} \frac{\partial}{\partial p} \frac{N^{e^{ \pm}}}{p^{2}}= \\
& =Q^{e^{ \pm}}(p, r, z)+c \beta n_{\mathrm{gas}}(r, z) \sigma_{e^{ \pm}} N^{\mathrm{CR}}
\end{aligned}
$$

Here $N^{e^{ \pm}}(p, r, z)$ is the number density of the electrons/positrons; $p$ is their momentum; $\beta$ their velocity in units of the speed of light $c ; \sigma_{e^{ \pm}}$is the production cross section of electrons/positrons from spallation of CR protons and heavier nuclei with density $N^{\mathrm{CR}}$ onto the ISM gas, whose density is $n_{\text {gas }} ; D=\beta D_{0}(\mathcal{R} / \mathrm{GV})^{\delta}$ is the spatial diffusion coefficient which is a function of the particle rigidity $\mathcal{R} ; \boldsymbol{v}_{c}$ is the convection velocity; $\dot{p}$ are continuous energy losses, whose relevant timescales $\tau=p / \dot{p}$ are shown in figure 1 . The last term on the l.h.s. of eq. (2.1) describes diffusive reacceleration of $\mathrm{CR}$ in the turbulent galactic magnetic field. In the quasi-liner theory the diffusion coefficient in momentum space $D_{p p}$ is related to the spatial diffusion coefficient by the relationship (see e.g. [36])

$$
D_{p p}=\frac{4}{3 \delta\left(4-\delta^{2}\right)(4-\delta)} \frac{v_{A}^{2} p^{2}}{D}
$$

where $v_{A}$ is the Alfvèn velocity. Finally, $Q^{e^{ \pm}}(p, r, z)$ represents the primary injection term.

The electron distribution can be computed numerically in the stationary limit $\partial N^{e^{ \pm}} / \partial t \equiv 0$ by using the methods of [41-43]. However, for this paper we have used our own propagation codes, which have strongly improved convergence behavior for mono-energetic injection spectra. From this distribution we will then compute the $\gamma$-ray spectra and maps produced by ICS and bremsstrahlung. 

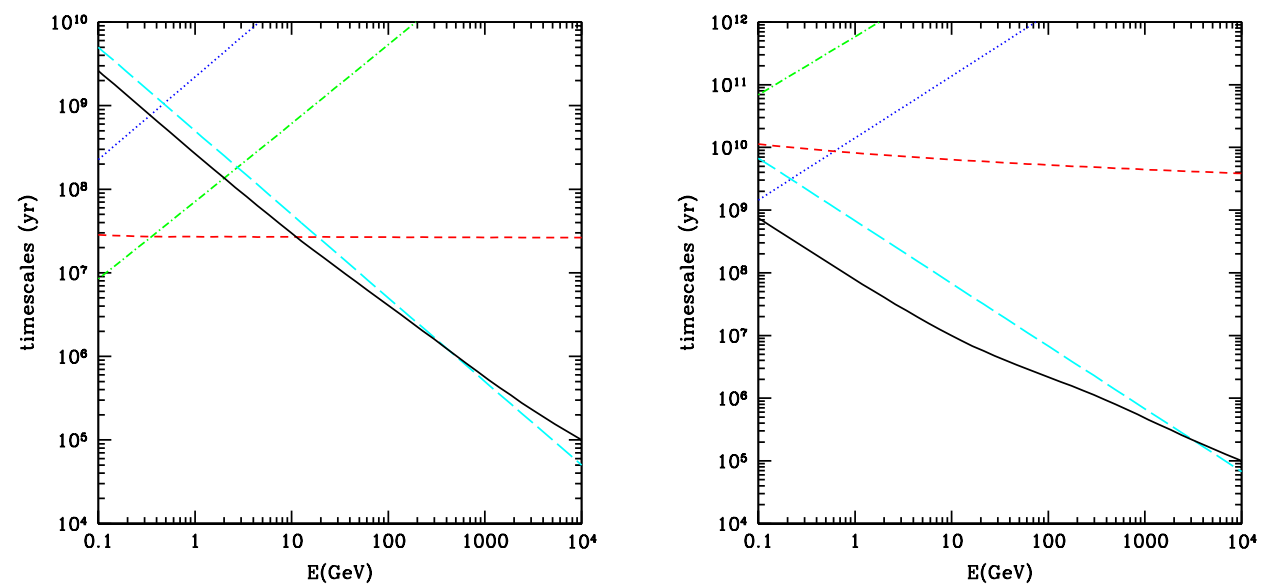

Figure 1. Energy loss time scales for CR electrons at Solar position (left panel) and at $r=0$ and $z=2 \mathrm{kpc}$ (right panel). The black solid curve represents IC losses, cyan long dashed is for synchrotron losses, red short dashed for bremsstrahlung losses, blue dotted for Coulomb losses, green dot-dashed for ionization losses.

We will firstly discuss inverse Compton scattering and bremsstrahlung radiation, and secondly prompt radiation, both with their galactic and extragalactic contributions, with particular emphasis on the related uncertainties.

\subsection{Inverse Compton scattering and bremsstrahlung}

Electrons and positrons produced in the decay of dark matter give rise to a $\gamma$-ray signal, coming from the inverse Compton scattering (ICS) of low energy photons of the interstellar radiation field (ISRF). A further, mostly subdominant, contribution to the $\gamma$-ray flux comes from bremsstrahlung of the electrons and positrons scattering off the galactic gas. In the context of the PAMELA positron excess, ICS radiation from decaying dark matter has been discussed in refs. [37, 38], for the case of annihilating dark matter see refs. [39, 40].

The ICS $\gamma$-ray flux due to injected mono-energetic electrons and positrons from dark matter decay inside of our Galaxy, and arriving on Earth from the direction $\hat{\Omega}$ (characterized by galactic latitude $b$ and longitude $l$ ), is given by

$$
J_{\mathrm{ICS}}\left(\hat{\Omega}, E_{\gamma} ; E_{e}\right)=\frac{1}{4 \pi} \int_{\text {l.o.s. }} d s \int_{m_{e}}^{\infty} d E P\left(E_{\gamma}, E\right) n_{e}\left(\mathbf{r}, E ; E_{e}\right),
$$

where $n_{e}\left(\mathbf{r}, E ; E_{e}\right)$ satisfies the stationary solutions to the propagation eq. (2.1) for a source term with monochromatic injection of positrons and electrons at an energy $E_{e}, P\left(E_{\gamma}, E\right)$ is the differential photon emissivity and the spatial integral is along the line of sight. The relevant source function is given by

$$
Q(\boldsymbol{r}, E)=\frac{\rho_{\chi}(\boldsymbol{r})}{\tau_{\chi} m_{\chi}} \delta\left(E-E_{e}\right),
$$

where $m_{\chi}$ is the DM particle mass and $\tau_{\chi}$ is its lifetime.

As dark matter profile $\rho_{\chi}(\mathbf{r})$ we take (assuming, as customary, spherical symmetry) the NFW profile [44]

$$
\rho_{\chi}^{\mathrm{NFW}}(r) \propto \frac{r}{r_{s}} \frac{1}{\left(1+r / r_{s}\right)^{2}},
$$


which we normalize to $\rho_{\odot}=0.36 \mathrm{GeV} / \mathrm{cm}^{3}$ at the position of the Sun, $r_{\odot}=8.5 \mathrm{kpc}$, and we adopt $r_{s}=20 \mathrm{kpc}$. Uncertainties from the halo profile will be discussed below.

The differential emissivity $P\left(E_{\gamma}, E\right)$ corresponds to two processes,

$$
P\left(E_{\gamma}, E\right)=P_{\mathrm{IC}}\left(E_{\gamma}, E\right)+P_{\text {bremss }}\left(E_{\gamma}, E\right) .
$$

The first term $P_{\mathrm{IC}}\left(E_{\gamma}, E\right)$ corresponds to inverse Compton scattering, which is derived by convolving the differential number density of target photons with the well known KleinNishina cross section. The second term, $P_{\text {bremss }}\left(E_{\gamma}, E\right)$, stems from the bremsstrahlung emission due to deflection of relativistic electrons and positrons in the electrostatic potential of interstellar gas atoms and molecules. Since this contribution is subdominant in the energy range we consider in this work, we will always refer to the ICS channel as our reference channel in the following. More details of the calculation can be found in [45].

The galactic radiation field in the galactic plane consists mainly of starlight at wavelength $\lambda \sim 1 \mu \mathrm{m}$, diffuse dust emission at $\lambda \sim 100 \mu \mathrm{m}$ and the CMB. Notice however that since most of the radiation (CMB aside) is produced in the galactic plane, the actual radiation fields at large latitudes are poorly known, and the computation of ICS radiation from high latitudes suffers from large uncertainties. In this work we will adopt the ISRF presented in ref. [47].

The predicted $\gamma$-ray flux crucially depends on the propagation model. We list in table 1 three different models characterized by different choices of the propagation parameters in eq. (2.1). By comparing the results obtained with these three models we will estimate the uncertainties associated to the poor knowledge of the propagation models. The MIN and MAX models are diffusion-reacceleration-convection models. They were proposed in [48-50] and are known to be compatible with the observed $\mathrm{B} / \mathrm{C}$ ratio and produce the minimum and maximum observationally allowed fluxes of antiprotons on Earth, respectively. The L1 model is derived from [51], in which an energy dependent analysis of recent data about secondary/primary ratios allowed a fairly accurate study of the diffusion parameters $\delta$ and $D_{0} / L{ }^{1}$ Although the main aim of [51] was not to find a best fit model able to reproduce all the observed spectra, it is remarkable that the diffusion parameters determined via an high energy analysis are able to describe data down to energies of the order of $1 \mathrm{GeV} /$ nucleon, and also to reproduce with reasonable accuracy the PAMELA measurements of the antiproton flux. It must be noticed, however, that this is only one out of many different possible models in agreement with nuclei CR observations (also simple leaky-box models can in fact succeed in reproducing high energy data [52]). In fact, all three models listed in table 1 well reproduce available data, in spite of the different parameters they adopt. Moreover, all these models assume cylindrical symmetry and diffusion to be homogeneous and uniform over the whole Galaxy, which is a rough approximation of the galactic propagation regime. Yet, this simple modeling allows to reasonably reproduce available data with relatively few free parameters. A study of the effects of a possible spatial dependence of the diffusion coefficient in a general three-dimensional geometry is beyond the scope of this work.

For illustrative purposes, we show in figure 2 the predicted $\gamma$-ray emission for the L1 model of table 1 at $0.1,1$ and $10 \mathrm{GeV}$, respectively, assuming a dark matter decay into $e^{+} e^{-}$,

\footnotetext{
${ }^{1}$ It is a general fact that stable secondary/primary ratios do not allow to probe separately the magnitude of the diffusion coefficient and the height of the diffusion region. Unstable/stable ratios, such as the ${ }^{10} \mathrm{Be} /{ }^{9} \mathrm{Be}$, can in principle provide such a discrimination. The available data on such ratios, however, have very large errors, so that it is extremely difficult to extract information from them.

${ }^{2}$ Below/above the break in rigidity at $\mathcal{R}=4 \mathrm{GV}$ for the MIN, MAX and L1 model.
} 


\begin{tabular}{|c|c|c|c|c|c|c|c|c|}
\hline Model & $\delta^{2}$ & $\begin{array}{c}D_{0} \\
{\left[10^{28} \mathrm{~cm}^{2} / \mathrm{s}\right]}\end{array}$ & $\begin{array}{c}R \\
{[\mathrm{kpc}]}\end{array}$ & $\begin{array}{c}L \\
{[\mathrm{kpc}]}\end{array}$ & $\begin{array}{c}V_{c} \\
{[\mathrm{~km} / \mathrm{s}]}\end{array}$ & $\begin{array}{c}d V_{c} / d z \\
\mathrm{~km} / \mathrm{s} / \mathrm{kpc}\end{array}$ & $\begin{array}{c}V_{a} \\
{[\mathrm{~km} / \mathrm{s}]}\end{array}$ & $\begin{array}{c}h_{\text {reac }} \\
{[\mathrm{kpc}]}\end{array}$ \\
\hline MIN & $0.85 / 0.85$ & 0.048 & 20 & 1 & 13.5 & 0 & 22.4 & 0.1 \\
L1 & $0.50 / 0.50$ & 4.6 & 20 & 4 & 0 & 0 & 10 & 4 \\
MAX & $0.46 / 0.46$ & 2.31 & 20 & 15 & 5 & 0 & 117.6 & 0.1 \\
\hline
\end{tabular}

Table 1. Typical combinations of diffusion parameters that are consistent with an analysis of CR nuclei secondary/primary ratios. The MIN and MAX propagation models correspond to minimal and maximal primary antiproton fluxes, respectively, while the L1 model can provide a good description of $\mathrm{B} / \mathrm{C}, \bar{p} / p$ and data on other secondary/primary ratios above $1 \mathrm{GeV} / \mathrm{n}$.
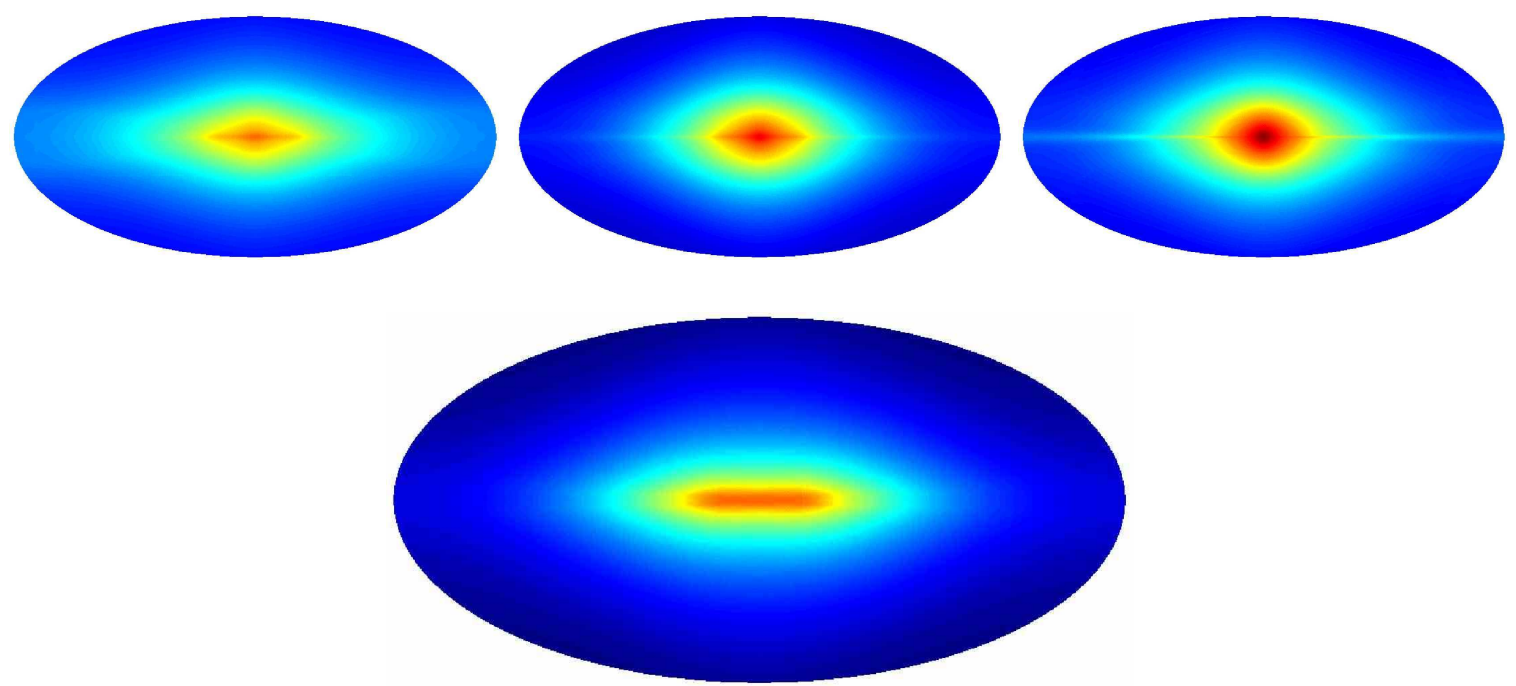

$-7.18$

Figure 2. The $\gamma$-ray emissions at $0.1 \mathrm{GeV}, 1 \mathrm{GeV}$ and $10 \mathrm{GeV}$ (upper panel from left to right) produced by dark matter particles decaying into $e^{+} e^{-}$pairs, where $m_{\chi}=200 \mathrm{GeV}, \tau_{\chi}=10^{26} \mathrm{~s}$. Results hold for the L1 diffusion model of table 1 and for the NFW halo profile. The lower panel shows the ICS radiation from astrophysical sources at $10 \mathrm{GeV}$ for comparison (again from model L1). The color scaling corresponds to the logarithm to the base 10 of the flux in $\mathrm{GeV} / \mathrm{s} / \mathrm{cm}^{2} / \mathrm{sr}$. Note that the color scale corresponds to the same flux range in all panels.

where $m_{\chi}=200 \mathrm{GeV}$ and $\tau_{\chi}=10^{26} \mathrm{~s}$. We also show for comparison the ICS radiation from primary electrons of astrophysical origin. In general, as apparent from these plots, dark matter induced ICS radiation extends to higher latitudes than the ICS radiation due to the contribution of standard astrophysical source, which is mainly concentrated on the galactic disk.

In addition to the ICS radiation produced in our Galaxy there is also a related extragalactic contribution, resulting from scattering of electrons from dark matter decaying outside of our Galaxy with the CMB. This component can potentially dominate the overall ICS fluxes at low energies, and we include it for completeness. The calculation is straightforward and details can be found in, e.g., ref. [37, 40]. In contrast to these references, we also took into account absorption effects due to inelastic scattering between ICS photons and the intergalactic background light (IBL), following ref. [38, 53] (adopting the "fast evolution" model).

Another contribution to the ICS radiation comes from electrons and positrons produced in the dark matter decay inside of our dark matter halo, but outside of the diffusion zone. 
If these particles are far enough from the galactic disk region, beyond a few kpc, their main energy loss channel is scattering with the CMB. The corresponding propagation length is $\mathcal{O}(100 \mathrm{kpc})$ for $1 \mathrm{TeV}$ particles, and $\mathcal{O}(10 \mathrm{kpc})$ for $10 \mathrm{TeV}$ particles. Calculating the corresponding ICS flux while neglecting the motion of the particles in general overestimates this flux by an $\mathcal{O}(1)$ factor. Being conservative we do not include this radiation component to our bounds and leave a more careful calculation for future work.

Uncertainties. The largest uncertainties in the predicted $\gamma$-ray fluxes come from poorly known propagation parameters, in particular from the height of the diffusion zone. The corresponding uncertainties can reach one order of magnitude.

As we already noticed, the height $L$ is only poorly known since its determination is degenerate with the diffusion coefficient. The most widely adopted range of variation of $L$ is between 1 and $15 \mathrm{kpc}$, based on the poor quality data on ${ }^{10} \mathrm{Be} /{ }^{9} \mathrm{Be}$. Due to this uncertainty it is hard to obtain any definitive constraint on, e.g., the supersymmetric parameter space of DM based on current anti-proton data [49, 54]. The forthcoming AMS-02 experiment [55] will however provide very accurate data on unstable/stable ratios (as well as for B/C and other stable secondary/primary ratios), which might allow a more precise determination of the diffusion height scale $L$.

In our case it is clear that a larger height of the diffusion zone leads to more DM decays contributing to the $\gamma$-ray flux, because more electrons of DM origin are confined in the diffusive region. Uncertainties on other $\mathrm{CR}$ propagation parameters, such as the Alfvèn velocity $v_{A}$ and the convection velocity $v_{c}$, are less relevant in affecting the electron distribution of DM, and especially affect only the electrons below around $10 \mathrm{GeV}$ since higher energy electrons lose energy too rapidly via ICS and can not propagate over long distances.

We found that the full-sky ICS $\gamma$-ray emission induced by very high energy electrons from DM decay obtained in the L1 model is comparable with the one obtained using other widely known models, namely DC and DR [41] which adopt the same height of the diffusion zone as our L1 model.

Another source of uncertainty comes from the dependence of the $\gamma$-ray emission on the halo profiles. We compared the fluxes predicted for different halo models (with parameters as in ref. [38]) and found that for shallower halo profiles such as the Kra [56] and the isothermal [57] profile the $\gamma$-ray emission is reduced by around $10 \%$. On the other hand, in case of the Einasto profile (see [3] and references therein) the flux is enhanced by $30 \%$. Since this variation is subdominant when compared with the uncertainties of the propagation models, we will simply adopt the NFW halo profile in the rest of the present work.

The $\gamma$-ray emission also depends on the Galactic magnetic field, since synchrotron losses can be of the order of ICS radiation losses at high electron energies. The magnetic field profile close to the Galactic center is quite uncertain and could be considerably higher than a few $\mu \mathrm{G}$ [59]. In this paper, we adopt the field from ref. [58] which matches the 408 $\mathrm{MHz}$ synchrotron distribution. We compared our results with another widely used magnetic field and found that the $\gamma$-ray fluxes at high energies increase by just $15 \%$ by changing the magnetic field model to the one presented in ref. [60] which is based on a large-scale data set on starlight polarization.

\subsection{Prompt radiation}

Although this work focuses on ICS radiation produced by electrons and positrons from decaying dark matter, we cannot overlook the fact that the prompt radiation of gamma rays 
can be a very competitive signature of dark matter decays. Indeed, in many realistic cases it turns out that this component can be larger than the ICS and therefore it can actually give the strongest constraints.

Prompt radiation from dark matter decay is produced more frequently inside our overdense Galactic dark matter halo ${ }^{2}$ but it is also produced at cosmological distances. At energies around $10 \mathrm{GeV}$ or below, the magnitude of the halo and extragalactic fluxes are of the same order when looking in direction of the anti-galactic center, whereas at higher energies around and above $100 \mathrm{GeV}$ the inelastic scattering between $\gamma$ rays and the IBL reduces the extragalactic component significantly and can not be neglected.

We include the galactic and the extragalactic prompt radiation, following the calculations outlined in ref. [38]. Uncertainties come mainly from the adopted dark matter halo profile and its normalization at position of the Sun.

\section{$3 \quad e^{ \pm}$-response functions from inverse Compton scattering}

In this section we derive the $e^{ \pm}$-response functions for the $\gamma$-ray emission induced by DM decays into electrons and positrons by comparing our predictions with the 1-year observations of Fermi LAT in the energy range of $0.5 \mathrm{GeV}$ to $300 \mathrm{GeV}$.

As data we use the Fermi LAT $\gamma$-ray maps as derived in ref. [61]. ${ }^{3}$ In this analysis the events were binned into the eight energy ranges $0.5-1 \mathrm{GeV}, 1-2 \mathrm{GeV}, 2-5 \mathrm{GeV}, 5-10 \mathrm{GeV}$, 10-20 GeV, $20-50 \mathrm{GeV}, 50-100 \mathrm{GeV}$ and $100-300 \mathrm{GeV}$.

A few words of caution are required concerning the data basis: The adopted $\gamma$-ray maps are based on the "diffuse" event class, which at energies above 50 or $100 \mathrm{GeV}$ suffers from background contamination [62] that becomes relevant when diffuse fluxes are studied. In the highest energy regime the background contamination might be on the $50-80 \%$ level. Furthermore, no attempt of subtracting point sources were made. The adopted statistical errors are always derived from the exposure $3 \times 10^{10} \mathrm{~cm}^{2} \mathrm{~s}$, which is good enough for the purpose of this paper. ${ }^{4}$ We do not attempt to include systematic errors in the analysis, which in light of the large background contamination of the data would be too premature.

\subsection{Definitions and optimal sky patch}

Following the same Ansatz we made for the case of synchrotron radiation proposed in ref. [34], we introduce response functions, which are functions of the electron injection energy and are associated to $\gamma$-ray observations in a sky patch $\Delta \Omega$ and in an energy band $E_{0} \leq E_{\gamma} \leq E_{1}$. The $e^{ \pm}$-response functions are defined as the ratio of the predicted $\gamma$-ray fluxes resulting from decaying dark matter to the observed fluxes as

$$
F_{\gamma}^{E_{0}: E_{1}}\left(\Delta \Omega ; E_{e}\right) \equiv \frac{\int_{E_{0}}^{E_{1}} d E_{\gamma} \int_{\Delta \Omega} d \Omega J_{\mathrm{ICS}}\left(\Omega, E_{\gamma} ; E_{e}\right)}{J_{\mathrm{obs}}^{E_{0}: E_{1}}(\Delta \Omega)+2 \cdot \delta J_{\mathrm{obs}}}\left(\frac{\tau_{\chi}}{10^{26} \mathrm{~s}}\right)\left(\frac{m_{\chi}}{100 \mathrm{GeV}}\right)
$$

\footnotetext{
${ }^{2}$ Although the halo profile is expected to be approximately isotropic, the corresponding flux at Earth exhibits a strong dipole-like anisotropy due to the offset between the Sun and the galactic center, which can be used to distinguish it from the extragalactic $\gamma$-ray background, see ref. [38].

${ }^{3} \mathrm{We}$ also performed an analysis of the publicly available event data on http://fermi.gsfc.nasa.gov/ssc/data/, coming to identical results as ref. [61]. We decided to use the maps from [61] in order to keep the data basis of our analysis easily accessible.

${ }^{4}$ We cross-checked with our own analysis of the publicly available event data that this gives indeed the correct number counts at high energies.
} 
where $J_{\mathrm{ICS}}\left(\Omega, E_{\gamma} ; E_{e}\right)$ is calculated from the injection spectrum eq. (2.4) and we adopt the conservative attitude of adding the $2 \sigma$ error to the central value. These functions depend on neither $\tau_{\chi}$ nor $m_{\chi}$ of eq. (2.4), and constraints on a given DM decay model can then be easily cast in the form

$$
\int_{m_{e}}^{m_{\chi}} d E_{e} F_{\gamma}^{E_{0}: E_{1}}\left(\Delta \Omega ; E_{e}\right) \frac{d N_{e}}{d E_{e}} \leq\left(\frac{\tau_{\chi}}{10^{26} \mathrm{~s}}\right)\left(\frac{m_{\chi}}{100 \mathrm{GeV}}\right),
$$

where $d N_{e} / d E_{e}$ is the electron/positron spectrum obtained from DM decay in some specific particle physics model. We stress again that the advantage of the $e^{ \pm}$-response function approach is that the $e^{ \pm}$-response functions are independent of the specific DM decay spectrum. The method is hence directly applicable to any DM model, and, moreover, allows a discussion of the typical characteristics of ICS radiation from DM decay in a model-independent way.

The $e^{ \pm}$-response functions depend crucially on the chosen patch $\Delta \Omega$, which in the ideal case should cover the area with the largest signal-to-background ratio, maximizing the $e^{ \pm}$-response functions. For the sake of clarity of our approach, and to avoid problems with statistical bias related to adaptive methods (which are related to downward statistical fluctuations and become severe if the statistics is low, see ref. [63]), we choose a fixed patch with large signal-to-background ratio by inspection of the signal-to-background maps.

Clearly, the optimal region might in principle depend on the observed energy range and injection energy of electrons and positrons. But it turns out that for injection energies around $100 \mathrm{GeV}-10 \mathrm{TeV}$ and high enough $\gamma$-ray energies, the optimal region is always located at south of the galactic center. This situation is illustrated in figure 3, which shows an example of a signal-to-background map for observed energies between 0.5 and $1 \mathrm{GeV}$, where the statistics is very good, for dark matter decay producing monochromatic electrons and positrons at $100 \mathrm{GeV}$. The map already suggests that the location of the optimal patch for constraining inverse Compton light from decaying dark matter actually lies in a region close to the galactic center, located at south of the galactic plane.

Before discussing this further we note that an exception occurs for very high injection energies in the $1-10 \mathrm{TeV}$ region, and low enough observed $\gamma$-ray energies. There the overall ICS flux can actually be dominated by extragalactic ICS contributions from scattering between electrons and positrons from dark matter decay off the CMB. In these cases the optimal patch would be located at the pole regions. The same holds in general true for prompt radiation from dark matter decay in and outside of the Galaxy, which has a much shallower angular profile than the galactic ICS component.

A more quantitative description of the situation can be found in the plots shown in figure 4. The black lines in the upper four panels show the signal-to-background ratio in different observed energy ranges as function of galactic latitude or longitude. The injection energy is now fixed to $1 \mathrm{TeV}$, but the results stay qualitatively the same for other injection energies. As expected from the above discussion, at high energies (upper panels) the signal-to-background ratio is maximal in a region close to the galactic center, whereas at very low energies (middle panels) it is maximal at high latitudes, due to the extragalactic ICS component. The same dominance at high latitudes is also present in the case of prompt radiation (lower panels).

For the derivation of the $e^{ \pm}$-response functions we will concentrate on the patch $\mathcal{S}$ close to the galactic center and defined by $|l|<20^{\circ}$ and $-18^{\circ}<b<-10^{\circ}$, which is marked by the colored region in figure 4 . We checked that this patch indeed maximizes the obtained constraints when varying the patch boundaries, except for the very highest energy region $(100 \mathrm{GeV}-300 \mathrm{GeV})$, where however the statistical error is large. For this patch, we construct 


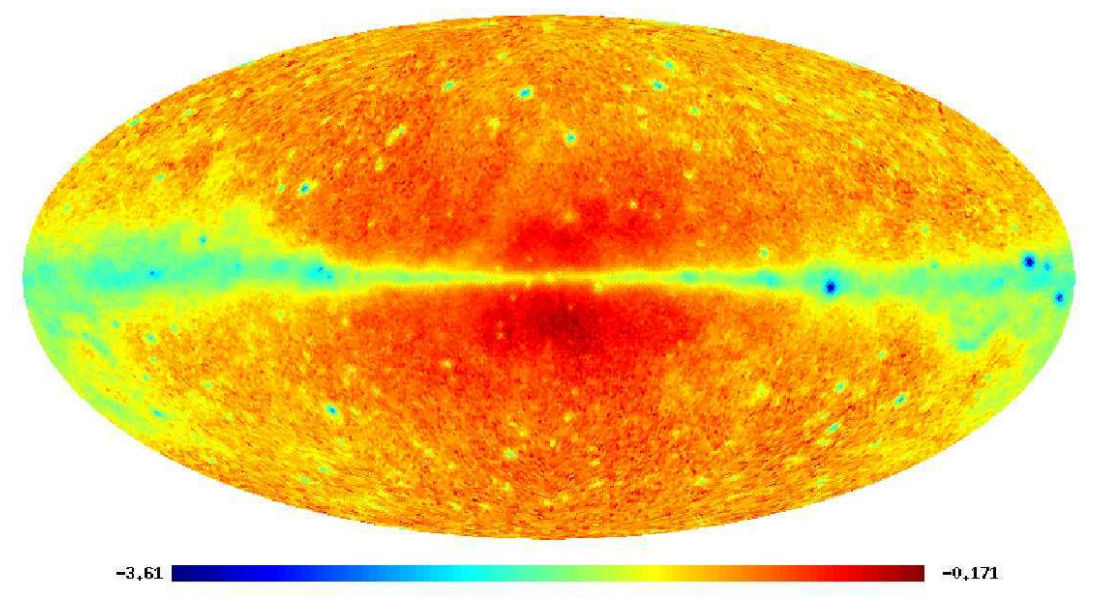

Figure 3. Signal-to-background map of ICS radiation from dark matter with $m_{\chi}=200 \mathrm{GeV}, \tau_{\chi}=$ $10^{26} \mathrm{~s}$ decaying into $e^{ \pm}$-pairs, compared to the Fermi LAT $\gamma$-ray observations in the $0.5-1 \mathrm{GeV}$ regime. Results hold for the L1 propagation model of table 1. Note the logarithmic color scaling, warmer colors indicate larger signal-to-background.

the $e^{ \pm}$-response functions by performing simulations injecting different mono-energetic electrons and comparing the results with observations according to eq. (3.1).

Given that in some cases the optimal patch is actually located at the galactic pole regions we will also calculate and present bounds that come from comparing the preliminary extragalactic $\gamma$-ray background as determined in ref. [62] with the extragalactic ICS and isotropic prompt radiation component, see below.

\subsection{Response functions without foreground subtraction}

Our results for the $e^{ \pm}$-response function are shown in figure 5 as function of the electron/positron injection energy, for the 8 different energy ranges of Fermi LAT skymaps from ref. [61]. The highest energy range provides the strongest constraint on decaying dark matter with very hard electron/positron energy spectrum. However, for lower injection energies in the $100 \mathrm{GeV}-1 \mathrm{TeV}$ region, several energy ranges give actually roughly the same constraints.

To illustrate the large uncertainties related to inverse Compton radiation from dark matter decay inside the diffusive halo, we show in figure 6 the $e^{ \pm}$-response functions based on the highest and lowest $\gamma$-ray energy ranges for our three reference propagation models from table 1. As emphasized before, the uncertainties on the $e^{ \pm}$-response functions are dominated by the propagation model, especially for the lower energies, below $10 \mathrm{GeV}$ injection energy, where also effects of reacceleration become relevant. For higher injection energies above 10 $100 \mathrm{GeV}$, where the response functions become of $\mathcal{O}(1)$ and are hence relevant for the actual bounds, the uncertainties mainly stem from the height of the diffusion zone. In effect, high energy electrons and positrons lose energy in a very short time compared to the diffusion time, thus making the other details of the propagation irrelevant. The MAX propagation model gives the strongest constraints due to its large diffusive halo, whereas the MIN propagation model minimizes the constraints. Moreover, for the MAX model re-acceleration shifts lower energy electrons to higher energies. This effect is however only relevant for electrons below 

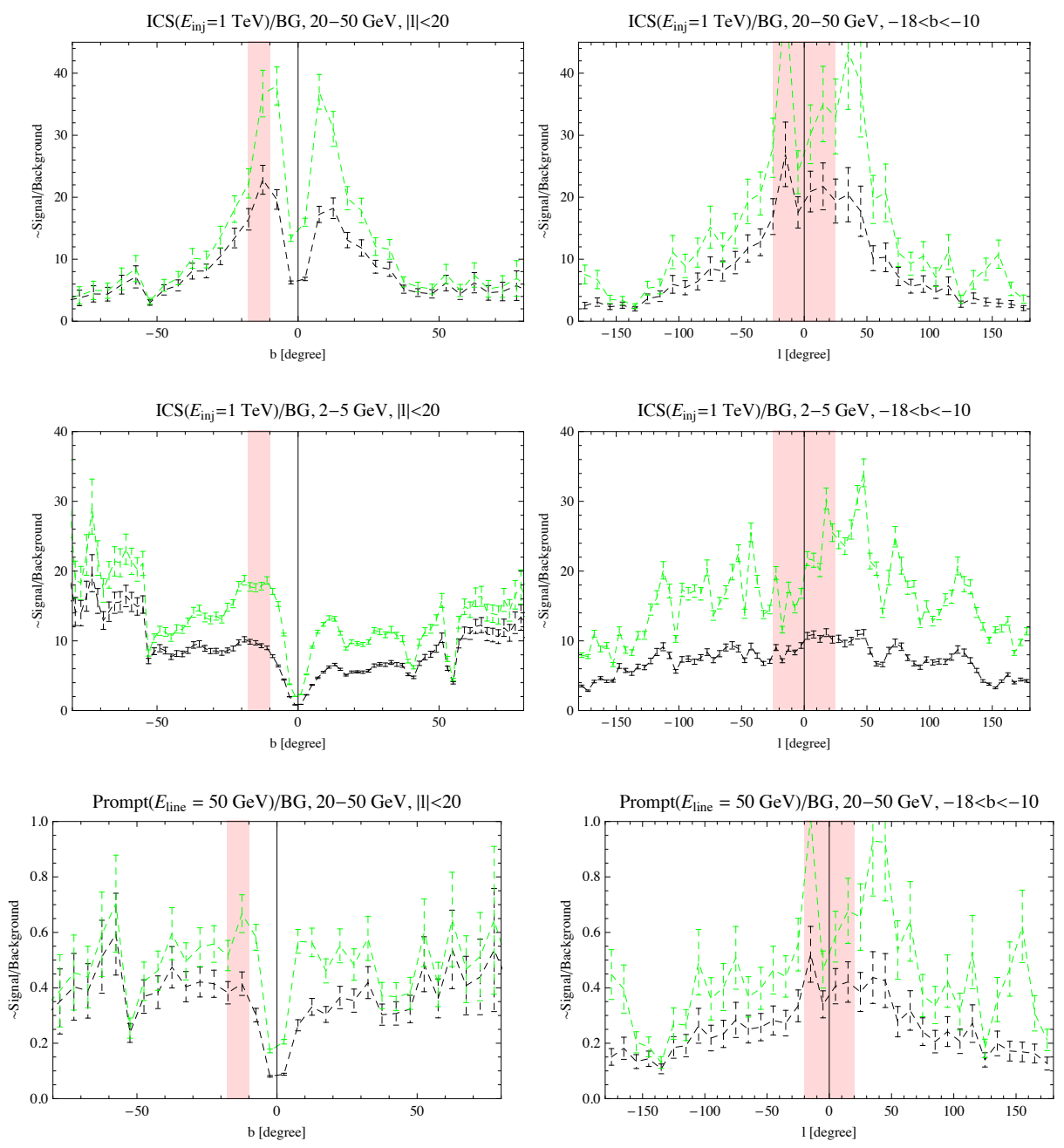

Figure 4. Signal-to-background ratios as function of galactic latitude (left panels) and longitude (right panels). The upper and middle panels correspond to pure ICS signal, the lower panels correspond to the pure prompt signal for comparison. Extragalactic and galactic radiation are taken into account. The black lines take into account as the whole observed signal, the green lines are obtained after subtraction of our reference model for the astrophysical component (Model L1). We find that the signal-to-background ratio of ICS radiation at higher $\gamma$-ray energies is maximized in the region $\mathcal{S}$ defined by $|l| \leq 20^{\circ}$ and $-18^{\circ} \leq b \leq-10^{\circ}$, which is indicated by the light red shaded region.

around $10 \mathrm{GeV}$, and thus increases the $\gamma$-ray emission only in the $\mathrm{MeV}$ regime. Note that for the highest observed $\gamma$-ray energy region $(100-300 \mathrm{GeV})$, one clearly finds a sharp cut off at low injection energies since $\gamma$-rays at such high energies cannot be produced from ICS of electrons/positrons injected at energies lower than $100 \mathrm{GeV}$.

\subsection{Response functions with subtraction of astrophysical foregrounds}

The $e^{ \pm}$-response functions discussed so far are conservative because we did not attempt to subtract any astrophysical contribution to the $\gamma$-ray flux. In order to understand the conventional astrophysical $\gamma$-ray flux one needs to estimate the $\gamma$-ray emission from different 


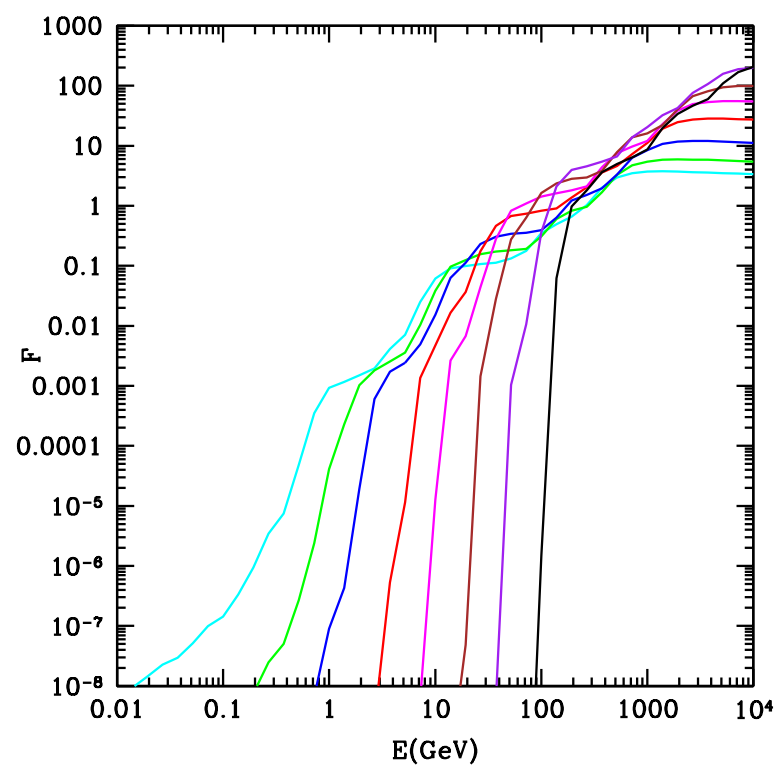

Figure 5. The $e^{ \pm}$-response function $F_{\gamma}$ based on $\gamma$-ray emission for the L1 model of table 1 . The $e^{ \pm}$-response functions are derived from the eight $\gamma$-ray energy ranges $0.5-1 \mathrm{GeV}, 1-2 \mathrm{GeV}, 2-5 \mathrm{GeV}$, 5-10 GeV, $10-20 \mathrm{GeV}, 20-50 \mathrm{GeV}, 50-100 \mathrm{GeV}$, and $100-300 \mathrm{GeV}$ from top to bottom at left side, respectively. The underlying sky patch $\mathcal{S}$ is defined by $|l| \leq 20^{\circ}$ and $-18^{\circ} \leq b \leq-10^{\circ}$.

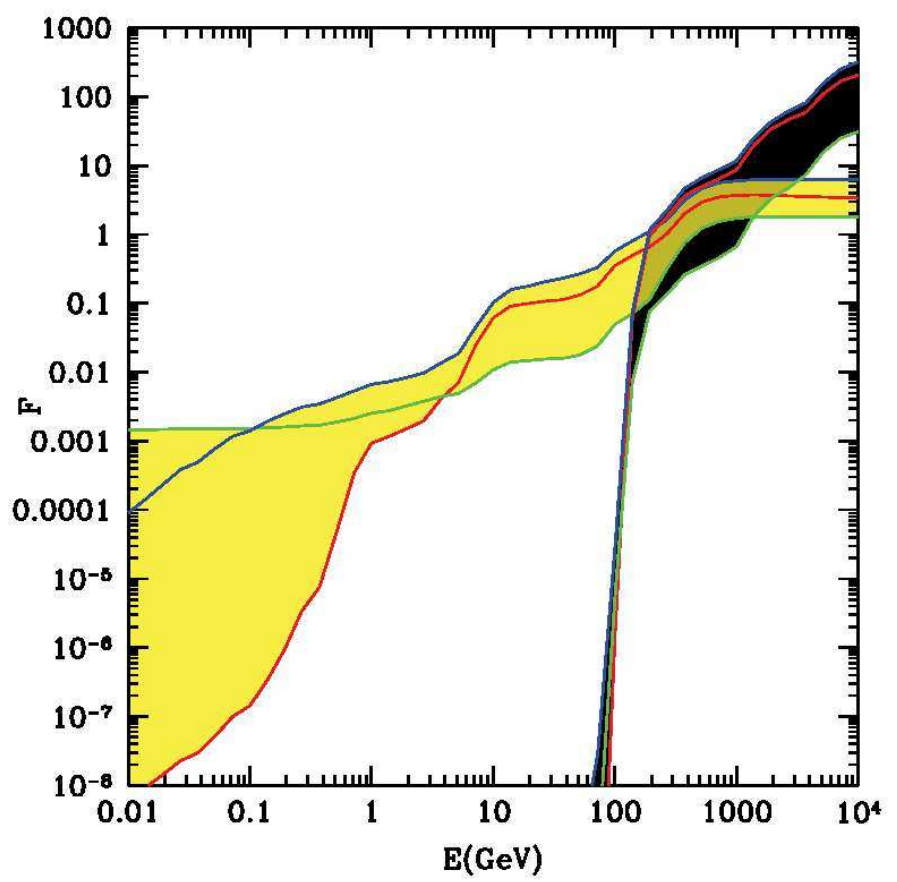

Figure 6. The propagation model dependence of the $e^{ \pm}$-response function $F_{\gamma}$ based on our fixed patch for the $\gamma$-ray energy range $0.5-1 \mathrm{GeV}$ (yellow band, curves extending to low energies) and $100-300 \mathrm{GeV}$ (black band, curves cutting off around $100 \mathrm{GeV}$ ). The width of the bands represents the variation within the MIN (green), L1 (red) and MAX (blue) propagation models of table 1. 


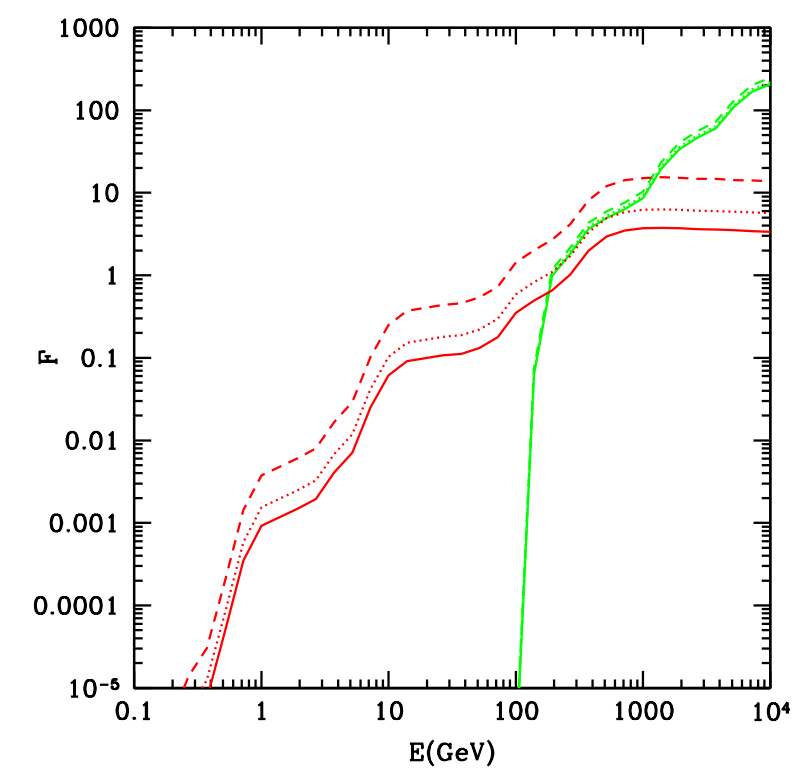

Figure 7. The dependence of the $e^{ \pm}$-response functions on subtraction of astrophysical contributions to the $\gamma$-ray signal. The $e^{ \pm}$-response function for the L1 model based on the raw observed map (solid) and on residual maps with $\gamma$-rays from $\pi^{0}$ decay (dotted) and from all astrophysical processes (dashed, see text) removed. Red lines extending below $1 \mathrm{GeV}$ are based on $\gamma$-ray flux observed in the energy range $0.5-1 \mathrm{GeV}$ and green lines are based on the interval $100-300 \mathrm{GeV}$.

galactic components. The most relevant production channels are nucleus-nucleus (mainly proton-proton) photoproduction via $\pi^{0}$ decay and ICS and bremsstrahlung of CR electrons and positrons. It is generally found [58] that hadronically generated $\gamma$-rays dominate the flux at energies between 0.1 and $100 \mathrm{GeV}$ and in the vicinity of the galactic plane, where most of the interstellar gas is located, while at lower and higher energies and at high latitudes ICS becomes comparable and can dominate. Bremsstrahlung is usually a subdominant component.

In figure 7 we show the $e^{ \pm}$-response function for the L1 model based on residual $\gamma$-ray maps obtained by subtracting $\gamma$-rays produced via $\pi^{0}$ decay, ICS and bremsstrahlung. In this foreground model the electron flux is adjusted to always lie below the electron flux observed by Fermi LAT, with a spectral index of around -3.2. The subtraction affects the results at low energies. For example, at $E_{e} \sim 10 \mathrm{TeV}$ the $e^{ \pm}$-response functions based on $\gamma$-ray fluxes observed at energies $0.5-1 \mathrm{GeV}$ are increased by a factor of around five when subtracting the total astrophysical "foreground" at these $\gamma$-ray energies. Again at $E_{e} \sim 10 \mathrm{TeV}$ the $e^{ \pm}$-response functions based on $\gamma$-ray fluxes observed at energies $100-300 \mathrm{GeV}$ are increased by $<10 \%$ and $\sim 15 \%$ by the removal of $\gamma$-rays originating from $\pi^{0}$ decay and from all astrophysical processes, respectively. This demonstrates explicitly that constraints on dark matter decay can be improved by taking into account the removal of astrophysical contributions mentioned above. At high energies the removal turns out to be quite insufficient which is at least in part related to the strong background contamination in the adopted data. This situation will be improved when data with better background rejection becomes available. 


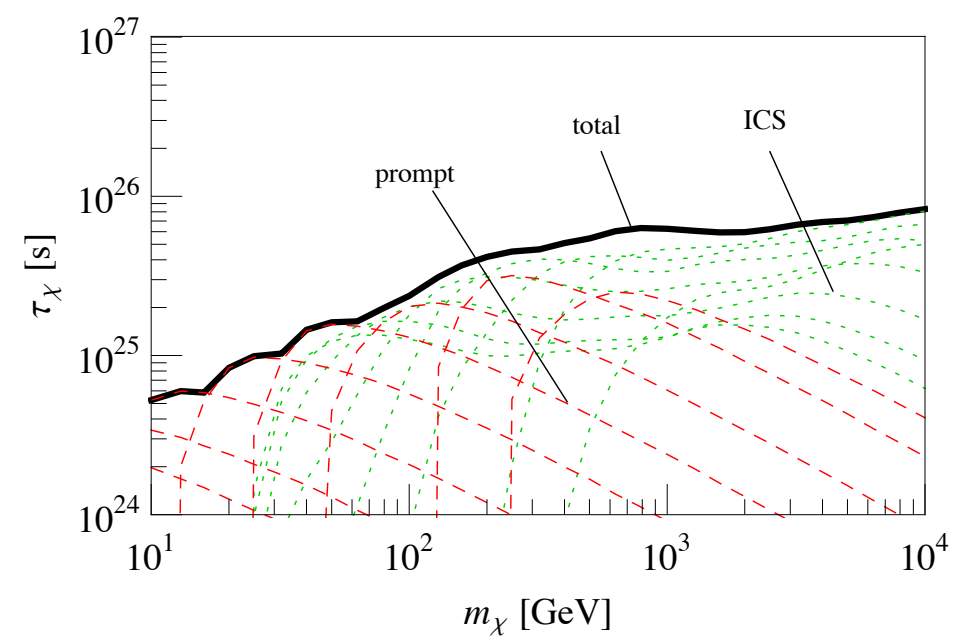

Figure 8. Constraints on decaying dark matter for the decay channel $\chi \rightarrow \mu^{+} \mu^{-}$decoded into its different components. The thick solid line shows the overall bounds on mass and lifetime, $c f$. also figure 9. Green lines represent the constraint coming from the $e^{ \pm}$-response function for ICS emission alone, whereas red lines are based on the prompt photon spectrum alone. Each of the eight lines corresponds to one of the observed $\gamma$-ray energy ranges as denoted in the caption of figure 5 .

\section{Constraints on dark matter models}

In the previous sections we constructed $e^{ \pm}$-response functions for DM decays which are independent of the particles physics details of the decay and only depend on the spatial distribution of the DM particles and the propagation of the produced electrons and positrons. These $e^{ \pm}$-response functions are very useful to estimate the constraints on DM decay based on $\gamma$-rays produced by ICS within some specific DM model. However, within a given DM model $\gamma$-rays can be produced not only as secondaries of electron and positron propagation in the Galaxy, but also as final-state, or prompt, radiation arising in the decay [64, 65]. For selected decay channels, we calculated the corresponding galactic and extragalactic prompt fluxes in our selected patch as described above and added them to the one described by the $e^{ \pm}$-response functions in order to derive constraints that come from the total prompt + ICS radiation flux of different dark matter decaying models. Other works on constraints on the decaying/annihilating dark matter interpretation of the PAMELA positrons excess with recent Fermi LAT $\gamma$-data can be found in ref. [24, 63, 66].

To illustrate the interplay of prompt and ICS radiation bounds, we show them in detail for the exemplary decay channel into $\mu^{+} \mu^{-}$in figure 8. In this plot, each of the green lines corresponds to bounds coming purely from the galactic and extragalactic ICS radiation (calculated from our response functions) for the eight different energy regimes of the data. Regions below the green lines are excluded. On the other hand, the red lines show the corresponding bounds when only prompt radiation is taken into account. The thick black line is obtained when both radiation components are combined for each energy regime separately. All fluxes are calculated within our patch $\mathcal{S}$. As obvious from this plot ICS radiation bounds dominate at dark matter masses above a few $100 \mathrm{GeV}$.

In figure 9 we show our results for bounds on the four different decay channels into $\mu^{+} \mu^{-} \mu^{+} \mu^{-}, \mu^{+} \mu^{-}, \tau^{+} \tau^{-}$and $b \bar{b}$ as examples with different amount of prompt and ICS 

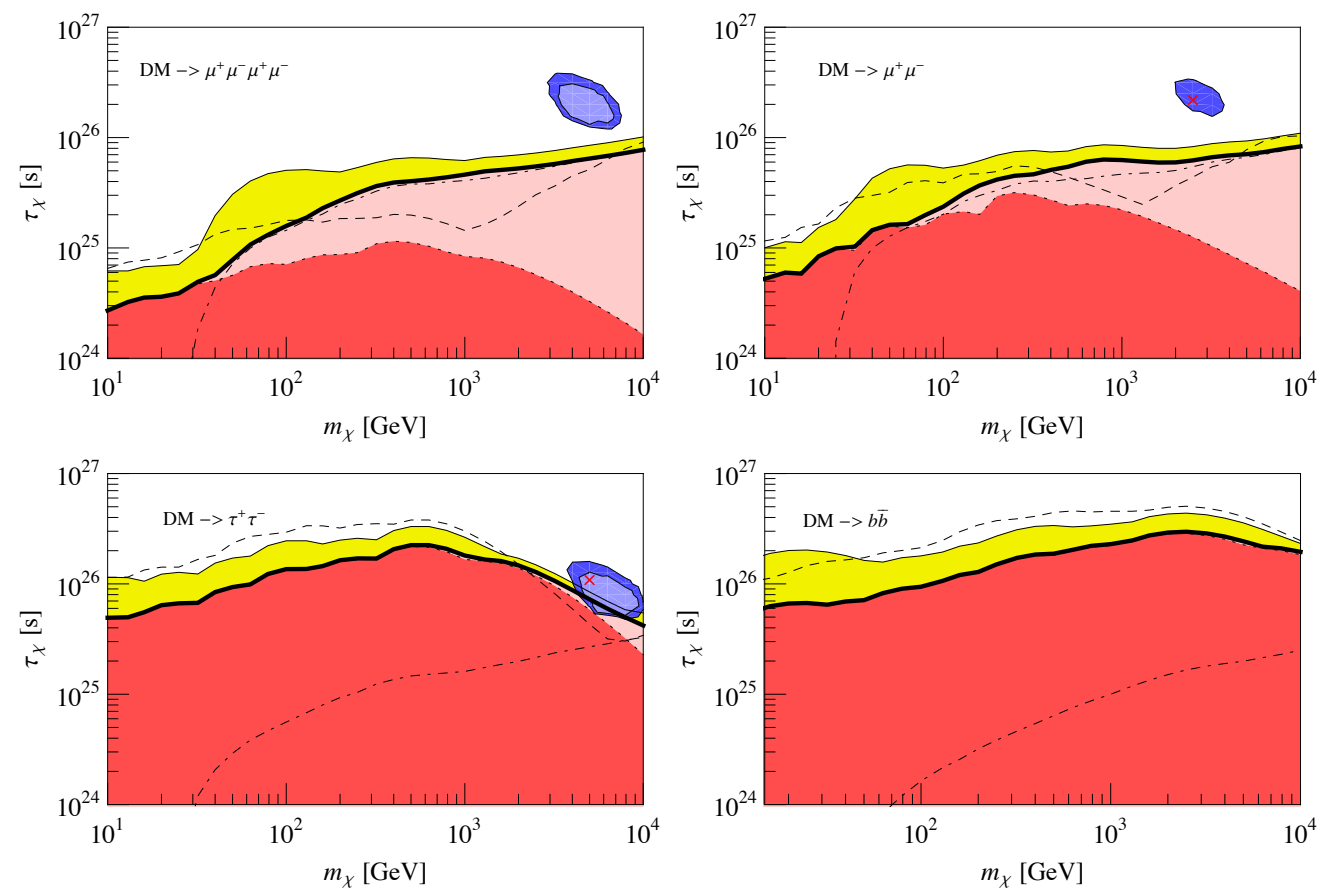

Figure 9. Bounds on different decay channels in the mass vs. lifetime plane. Regions below the thick solid line are excluded by combined ICS and prompt radiation in the L1 propagation model, whereas parameter space below the dashed-dotted (dashed) line is excluded due to ICS (prompt) radiation alone. The ICS constraints shown with the dashed-dotted lines are calculated from the response functions shown in figure 5. The constraints can be strengthened to the yellow light shaded region if the predictions of Model L1 for the galactic diffuse astrophysical foreground is subtracted. The blue blobs and red crosses (which are taken from ref. [21]) show the parameters that well fit electron + positron fluxes observed by Fermi LAT and HESS and the positron fraction observed by PAMELA as described in the text.

radiation. ${ }^{5}$ The first three decay modes can well fit the PAMELA/Fermi positron and electron data if the positron excess is interpreted in terms of decaying dark matter, and the preferred mass and lifetime regions are indicated by the blue blobs and red crosses. ${ }^{6}$ We used the PyTHIA package [67] to derive the electron, positron and $\gamma$-ray decay spectra.

In these plots the dashed-dotted (dotted) line shows the bounds obtained from ICS (prompt) radiation in our patch $\mathcal{S}$ alone, the thick solid line shows the bounds obtained when prompt and ICS radiation are combined. Furthermore, the bounds can be strengthened to the yellow region when the foreground model L1 is subtracted from the data.

\footnotetext{
${ }^{5}$ The four-body decay into muons proceeds via two intermediate neutral scalar particles with masses of $1 \mathrm{GeV}$.

${ }^{6}$ The shown regions should be understood as typical masses and lifetimes that well fit the data. We performed a $\chi^{2}$ test, keeping the electron background as a freely adjustable powerlaw with spectral index between -3.3 and -3.0, whereas the positron background is kept fixed as the one from "Model 0" in ref. [32]. We include in the fitting procedure only the PAMELA data on the positron fraction above $10 \mathrm{GeV}$, as they should be less affected by solar modulation, and the Fermi and HESS $e^{ \pm}$-data, for which we added the corresponding systematic and statistical errors in square. Since systematic errors are correlated, the obtained $\chi^{2}$ are relatively small, but in view of the large uncertainties in the electron background a more detailed fit is not reasonable. The blue regions correspond to $\chi^{2} /$ dof $=1$ and $\chi^{2} /$ dof $=0.75$, outside these regions the fits to HESS and Fermi LAT become problematic.
} 
It turns out that for decays into $\mu^{+} \mu^{-}$pairs and four-body decay into $\mu^{+} \mu^{-} \mu^{+} \mu-$, the strongest constraints typically come from ICS rather than from the prompt radiation and the constraints could be improved by more than a factor of 2 for small masses and by a few $10 \%$ for large masses after removal of the $\gamma$-ray emission from conventional astrophysical sources. In the case of decay into $\tau^{+} \tau^{-}$and $b \bar{b}$ the prompt radiation alone already provides strong constraints, which can again be improved by subtracting galactic foreground as for the case of decay into muons.

Note that our patch $\mathcal{S}$ is optimized for ICS radiation. Prompt radiation from dark matter decay in general dominates at the galactic pole regions, as discussed above (in the actual data, this behavior is disturbed at high gamma-ray energies because of the large contamination of the data with isotropic cosmic-ray background). Following the slicing of the sky as proposed in ref. [63], we can find for the highest energy bin a patch that actually increases our corresponding final state radiation bounds by around $70 \%{ }^{7}$

For comparison, we also show with the dashed lines in figure 9 the bounds that can be obtained by comparing the sum of extragalactic ICS radiation, extragalactic prompt radiation and the maximal isotropic part of the halo prompt radiation (which is identical to the flux from the Galactic anti-center) with the preliminary results for the isotropic extragalactic gamma-ray flux as presented in ref. [62]. Comparing these bounds, which already rely on a foreground subtraction, in case of decay into muons with the ones obtained from patch $\mathcal{S}$ after foreground subtraction shows that they are subdominant and become only relevant at very high masses. Our bounds are somewhat weaker than the ones found in ref. [66], which is due to our inclusion of absorption effects and our more conservative treatment of extragalactic ICS radiation.

\section{Conclusions}

In this study we have calculated the contribution to the $\gamma$-ray fluxes from decaying dark matter particles, including the inverse Compton photons resulting from energetic electrons and positrons through scattering off low energy target photons in addition to the bremsstrahlung emissions. We constructed $e^{ \pm}$-response functions based on the full-sky $\gamma$-ray observations by Fermi LAT which can be applied to constrain any decaying dark matter model by convolving it with the specific decay spectrum into electrons and positrons. We also studied the dependence of the $e^{ \pm}$-response functions on both the set of propagation parameters and halo profiles and find that the most important uncertainty comes from the height of the diffusion zone.

We applied the response functions to different decaying dark matter models with leptonic final states, including also the effects of prompt radiation, which can significantly increase the $\gamma$-ray emissions in the dark halo. Moreover, we demonstrated how the constraints can be further improved by subtracting astrophysical contributions to the observed $\gamma$-ray flux.

Under the conservative assumption of a propagation model with the height of the diffusion zone around $4 \mathrm{kpc}$, based on the Fermi LAT data, we can severely constrain but not exclude models with dark matter decay into $\tau^{+} \tau^{-}$that can explain the positron excess observed by PAMELA. Moreover, we find that analogous models with two- and four-body decay channels into $\mu^{ \pm}$S remain essentially unconstrained by current observations. When our reference foreground model is subtracted the lower bounds on the lifetime in general increase

\footnotetext{
${ }^{7}$ Using this adaptively determined patch, which is located at $10^{\circ} \leq b \leq 20^{\circ}$ and $0 \leq l \leq 10^{\circ}$ and has only a few number counts, still does not allow to raise the bounds as high as shown in ref. [63]. The difference might originate in the smaller energy bins used in [63], and the inclusion of data above $300 \mathrm{GeV}$.
} 
by $\mathcal{O}(1)$ factors for dark matter masses below $1 \mathrm{TeV}$, and by $10-60 \%$ for masses above $1 \mathrm{TeV}$, which is however not enough to exclude the above channels in the parameter regime relevant for PAMELA. The bounds might improve by $\mathcal{O}(1)$ factors when data with better background rejection is used. For comparison we also calculated conservative bounds from the isotropic extragalactic $\gamma$-ray background as inferred from the Fermi LAT data, finding again that even the decay into $\tau^{+} \tau^{-}$cannot be excluded in this way.

In this work we demonstrated the use of $e^{ \pm}$-response functions in constraining dark matter models. We plan to update the response functions as data with improved rejection of background becomes available.

\section{Acknowledgments}

This work was supported by the Deutsche Forschungsgemeinschaft through SFB 676 "Particles, Strings and the Early Universe: The Structure of Matter and Space-Time" and through GRK 602 "Future Developments in Particle Physics". LM acknowledges support from the State of Hamburg, through the Collaborative Research program "Connecting Particles with the Cosmos" within the framework of the LandesExzellenzInitiative (LEXI). CW thanks Alejandro Ibarra and David Tran for helpful discussions.

\section{References}

[1] G. Bertone, D. Hooper and J. Silk, Particle dark matter: evidence, candidates and constraints, Phys. Rept. 405 (2005) 279 [hep-ph/0404175] [SPIRES].

[2] P. Ullio, L. Bergstrom, J. Edsjo and C.G. Lacey, Cosmological dark matter annihilations into gamma-rays: a closer look, Phys. Rev. D 66 (2002) 123502 [astro-ph/0207125] [SPIRES].

[3] L. Pieri, J. Lavalle, G. Bertone and E. Branchini, Implications of high-resolution simulations on indirect dark matter searches, arXiv:0908.0195 [SPIRES].

[4] M. Regis and P. Ullio, Testing the dark matter interpretation of the PAMELA excess through measurements of the galactic diffuse emission, Phys. Rev. D 80 (2009) 043525 [arXiv:0904.4645] [SPIRES].

[5] J.M. Siegal-Gaskins, Revealing dark matter substructure with anisotropies in the diffuse gamma-ray background, JCAP 10 (2008) 040 [arXiv:0807.1328] [SPIRES];

S. Ando, Gamma-ray background anisotropy from galactic dark matter substructure, Phys. Rev. D 80 (2009) 023520 [arXiv:0903.4685] [SPIRES];

B.S. Hensley, J.M. Siegal-Gaskins and V. Pavlidou, The detectability of dark matter annihilation with Fermi using the anisotropy energy spectrum of the gamma-ray background, arXiv:0912.1854 [SPIRES].

[6] L. Zhang, X.-L. Chen, Y.-A. Lei and Z.-G. Si, The impacts of dark matter particle annihilation on recombination and the anisotropies of the cosmic microwave background, Phys. Rev. D 74 (2006) 103519 [astro-ph/0603425] [SPIRES];

L. Zhang and G. Sigl, Dark matter signatures in the anisotropic radio sky, JCAP 09 (2008) 027 [arXiv:0807.3429] [SPIRES];

Q. Yuan, B. Yue, X. Bi, X. Chen and X. Zhang, Leptonic dark matter annihilation in the evolving universe: constraints and implications, arXiv:0912.2504 [SPIRES].

[7] X.-L. Chen and M. Kamionkowski, Particle decays during the cosmic dark ages, Phys. Rev. D 70 (2004) 043502 [astro-ph/0310473] [SPIRES]. 
[8] L. Zhang, X. Chen, M. Kamionkowski, Z.-G. Si and Z. Zheng, Contraints on radiative dark-matter decay from the cosmic microwave background, Phys. Rev. D 76 (2007) 061301 [arXiv:0704.2444] [SPIRES].

[9] Y. Gong and X. Chen, Cosmological constraints on invisible decay of dark matter, Phys. Rev. D 77 (2008) 103511 [arXiv:0802.2296] [SPIRES].

[10] W. Buchmüller, L. Covi, K. Hamaguchi, A. Ibarra and T. Yanagida, Gravitino dark matter in R-parity breaking vacua, JHEP 03 (2007) 037 [hep-ph/0702184] [SPIRES];

G. Bertone, W. Buchmüller, L. Covi and A. Ibarra, Gamma-rays from decaying dark matter, JCAP 11 (2007) 003 [arXiv:0709.2299] [SPIRES];

A. Ibarra and D. Tran, Gamma ray spectrum from gravitino dark matter decay,

Phys. Rev. Lett. 100 (2008) 061301 [arXiv:0709.4593] [SPIRES];

A. Ibarra and D. Tran, Antimatter signatures of gravitino dark matter decay,

JCAP 07 (2008) 002 [arXiv:0804.4596] [SPIRES];

L. Covi, M. Grefe, A. Ibarra and D. Tran, Unstable gravitino dark matter and neutrino flux, JCAP 01 (2009) 029 [arXiv:0809.5030] [SPIRES];

W. Buchmüller, A. Ibarra, T. Shindou, F. Takayama and D. Tran, Probing gravitino dark matter, JCAP 09 (2009) 021 [arXiv:0906.1187] [SPIRES].

[11] A. Boyarsky, O. Ruchayskiy and M. Shaposhnikov, The role of sterile neutrinos in cosmology and astrophysics, Ann. Rev. Nucl. Part. Sci. 59 (2009) 191 [arXiv:0901.0011] [SPIRES].

[12] C.-R. Chen, F. Takahashi and T.T. Yanagida, Gamma rays and positrons from a decaying hidden gauge boson, Phys. Lett. B 671 (2009) 71 [arXiv:0809.0792] [SPIRES].

[13] A. Ibarra, A. Ringwald, D. Tran and C. Weniger, Cosmic rays from leptophilic dark matter decay via kinetic mixing, JCAP 08 (2009) 017 [arXiv: 0903.3625] [SPIRES];

A. Ibarra, A. Ringwald and C. Weniger, Hidden gauginos of an unbroken $\mathrm{U}(1)$ : cosmological constraints and phenomenological prospects, JCAP 01 (2009) 003 [arXiv:0809.3196] [SPIRES].

[14] A. Arvanitaki et al., Astrophysical probes of unification, Phys. Rev. D 79 (2009) 105022 [arXiv:0812.2075] [SPIRES].

[15] PAMELA collaboration, O. Adriani et al., An anomalous positron abundance in cosmic rays with energies 1.5-100 GeV, Nature 458 (2009) 607 [arXiv:0810.4995] [SPIRES].

[16] D. Hooper, P. Blasi and P.D. Serpico, Pulsars as the sources of high energy cosmic ray positrons, JCAP 01 (2009) 025 [arXiv:0810.1527] [SPIRES].

[17] H. Yuksel, M.D. Kistler and T. Stanev, TeV gamma rays from Geminga and the origin of the GeV positron excess, Phys. Rev. Lett. 103 (2009) 051101 [arXiv:0810.2784] [SPIRES].

[18] D. Malyshev, I. Cholis and J. Gelfand, Pulsars versus dark matter interpretation of ATIC/PAMELA, Phys. Rev. D 80 (2009) 063005 [arXiv:0903.1310] [SPIRES].

[19] S. Profumo, Dissecting Pamela (and ATIC) with Occam's razor: existing, well-known pulsars naturally account for the 'anomalous' cosmic-ray electron and positron data, arXiv:0812.4457 [SPIRES].

[20] T. Kobayashi, Y. Komori, K. Yoshida and J. Nishimura, The most likely sources of high energy cosmic-ray electrons in supernova remnants, Astrophys. J. 601 (2004) 340 [astro-ph/0308470] [SPIRES].

[21] A. Ibarra, D. Tran and C. Weniger, Decaying dark matter in light of the PAMELA and Fermi LAT data, JCAP 01 (2010) 009 [arXiv:0906.1571] [SPIRES].

[22] E. Nardi, F. Sannino and A. Strumia, Decaying dark matter can explain the electron/positron excesses, JCAP 01 (2009) 043 [arXiv:0811.4153] [SPIRES]; 
K. Hamaguchi, S. Shirai and T.T. Yanagida, Cosmic ray positron and electron excess from hidden-fermion dark matter decays, Phys. Lett. B 673 (2009) 247 [arXiv:0812.2374] [SPIRES];

B. Kyae, PAMELA/ATIC anomaly from the meta-stable extra dark matter component and the leptophilic Yukawa interaction, JCAP 07 (2009) 028 [arXiv:0902.0071] [SPIRES];

P.-F. Yin et al., PAMELA data and leptonically decaying dark matter,

Phys. Rev. D 79 (2009) 023512 [arXiv:0811.0176] [SPIRES];

K. Ishiwata, S. Matsumoto and T. Moroi, Cosmic-ray positron from superparticle dark matter and the PAMELA anomaly, Phys. Lett. B 675 (2009) 446 [arXiv:0811.0250] [SPIRES].

[23] A. Ibarra and D. Tran, Decaying dark matter and the PAMELA anomaly, JCAP 02 (2009) 021 [arXiv:0811.1555] [SPIRES];

C.-R. Chen, M.M. Nojiri, F. Takahashi and T.T. Yanagida, Decaying hidden gauge boson and the PAMELA and ATIC/PPB-BETS anomalies, Prog. Theor. Phys. 122 (2009) 553

[arXiv:0811.3357] [SPIRES];

$\mathrm{X}$. Chen, Decaying hidden dark matter in warped compactification, JCAP 09 (2009) 029 [arXiv:0902.0008] [SPIRES];

K.J. Bae and B. Kyae, PAMELA/ATIC anomaly from exotic mediated dark matter decay, JHEP 05 (2009) 102 [arXiv:0902.3578] [SPIRES];

N. Okada and T. Yamada, The PAMELA and Fermi signals from long-lived Kaluza-Klein dark matter, Phys. Rev. D 80 (2009) 075010 [arXiv:0905.2801] [SPIRES].

[24] C.-R. Chen, S.K. Mandal and F. Takahashi, Gamma-ray constraints on hadronic and leptonic activities of decaying dark matter, JCAP 01 (2010) 023 [arXiv:0910.2639] [SPIRES].

[25] N.J. Shaviv, E. Nakar and T. Piran, Natural explanation for the anomalous positron to electron ratio with supernova remnants as the sole cosmic ray source,

Phys. Rev. Lett. 103 (2009) 111302 [arXiv:0902.0376] [SPIRES].

[26] P. Blasi, The origin of the positron excess in cosmic rays, Phys. Rev. Lett. 103 (2009) 051104 [arXiv:0903.2794] [SPIRES].

[27] L. Stawarz, V. Petrosian and R.D. Blandford, On the energy spectra of GeV/TeV cosmic ray leptons, Astrophys. J. 710 (2010) 236 [arXiv:0908.1094] [SPIRES].

[28] R. Cowsik and B. Burch, On the positron fraction and the spectrum of the electronic component in cosmic rays, arXiv:0905.2136 [SPIRES].

[29] B. Katz, K. Blum and E. Waxman, What can we really learn from positron flux 'anomalies'?, arXiv:0907.1686 [SPIRES].

[30] FERMI-LAT collaboration, A.A. Abdo et al., Measurement of the cosmic ray $e^{+}$plus $e^{-}$ spectrum from $20 \mathrm{GeV}$ to $1 \mathrm{TeV}$ with the Fermi Large Area Telescope, Phys. Rev. Lett. 102 (2009) 181101 [arXiv:0905.0025] [SPIRES].

[31] H.E.S.S. collaboration, F. Aharonian et al., The energy spectrum of cosmic-ray electrons at TeV energies, Phys. Rev. Lett. 101 (2008) 261104 [arXiv:0811.3894] [SPIRES].

[32] FERMI-LAT collaboration, D. Grasso et al., On possible interpretations of the high energy electron-positron spectrum measured by the Fermi Large Area Telescope, Astropart. Phys. 32 (2009) 140 [arXiv:0905.0636] [SPIRES].

[33] FERMI-LAT collaboration, G. Di Bernardo, D. Gaggero and D. Grasso, The high energy cosmic ray electron spectrum measured by Fermi gamma-ray space telescope: some possible interpretations, arXiv:0912.3887 [SPIRES].

[34] L. Zhang, G. Sigl and J. Redondo, Galactic signatures of decaying dark matter, JCAP 09 (2009) 012 [arXiv:0905.4952] [SPIRES].

[35] V.L. Ginzburg and S.I. Syrovatskii, Proiskhozhdenie kosmicheskikh luchei (the origin of cosmic rays) (in Russian), USSR Academy of Sciences, Moscow Russia (1963) [Pergamon press, Oxford U.K. (1964)]. 
[36] E.S. Seo and V.S. Ptuskin, Stochastic reacceleration of cosmic rays in the interstellar medium, Astrophys. J. 431 (1994) 705S.

[37] K. Ishiwata, S. Matsumoto and T. Moroi, Cosmic gamma-ray from inverse Compton process in unstable dark matter scenario, Phys. Lett. B 679 (2009) 1 [arXiv:0905.4593] [SPIRES]; M. Pohl and D. Eichler, Fermi constrains dark matter origin of high energy positron anomaly, arXiv:0912.1203 [SPIRES];

K. Ishiwata, S. Matsumoto and T. Moroi, Cosmic gamma-ray from inverse Compton process in unstable dark matter scenario, AIP Conf. Proc. 1223 (2010) 60 [arXiv:0912.2486] [SPIRES].

[38] A. Ibarra, D. Tran and C. Weniger, Detecting gamma-ray anisotropies from decaying dark matter: prospects for Fermi LAT, Phys. Rev. D 81 (2010) 023529 [arXiv:0909.3514] [SPIRES].

[39] M. Cirelli and P. Panci, Inverse Compton constraints on the dark matter $e^{+} e^{-}$excesses, Nucl. Phys. B 821 (2009) 399 [arXiv:0904.3830] [SPIRES];

M.D. Kistler and J.M. Siegal-Gaskins, Gamma-ray signatures of annihilation to charged leptons in dark matter substructure, Phys. Rev. D 81 (2010) 103521 [arXiv: 0909.0519] [SPIRES];

J. Zhang et al., Discriminating different scenarios to account for the cosmic $e^{ \pm}$excess by synchrotron and inverse Compton radiation, Phys. Rev. D 80 (2009) 023007 [arXiv:0812.0522] [SPIRES];

E. Borriello, A. Cuoco and G. Miele, Secondary radiation from the Pamela/ATIC excess and relevance for Fermi, Astrophys. J. 699 (2009) L59 [arXiv: 0903.1852] [SPIRES].

[40] S. Profumo and T.E. Jeltema, Extragalactic inverse Compton light from dark matter annihilation and the Pamela positron excess, JCAP 07 (2009) 020 [arXiv:0906.0001] [SPIRES].

[41] A.W. Strong and I.V. Moskalenko, Propagation of cosmic-ray nucleons in the galaxy, Astrophys. J. 509 (1998) 212 [astro-ph/9807150] [SPIRES].

[42] I.V. Moskalenko and A.W. Strong, Production and propagation of cosmic-ray positrons and electrons, Astrophys. J. 493 (1998) 694 [astro-ph/9710124] [SPIRES].

[43] C. Evoli, D. Gaggero, D. Grasso and L. Maccione, Cosmic-ray nuclei, antiprotons and gamma-rays in the galaxy: a new diffusion model, JCAP 10 (2008) 018 [arXiv:0807.4730] [SPIRES].

[44] J.F. Navarro, C.S. Frenk and S.D.M. White, The structure of cold dark matter halos, Astrophys. J. 462 (1996) 563 [astro-ph/9508025] [SPIRES].

[45] G.R. Blumenthal and R.J. Gould, Bremsstrahlung, synchrotron radiation, and Compton scattering of high-energy electrons traversing dilute gases, Rev. Mod. Phys. 42 (1970) 237 [SPIRES].

[46] G.B. Rybicki and A.P. Lightman, Radiative processes in astrophysics, John Wiley \& Sons, U.S.A. (1979).

[47] T.A. Porter and A.W. Strong, A new estimate of the galactic interstellar radiation field between 0.1 microns and 1000 microns, astro-ph/0507119 [SPIRES].

[48] F. Donato et al., Antiprotons from spallation of cosmic rays on interstellar matter, Astrophys. J. 563 (2001) 172 [astro-ph/0103150] [SPIRES].

[49] F. Donato, N. Fornengo, D. Maurin and P. Salati, Antiprotons in cosmic rays from neutralino annihilation, Phys. Rev. D 69 (2004) 063501 [astro-ph/0306207] [SPIRES].

[50] D. Maurin, R. Taillet and F. Donato, New results on source and diffusion spectral features of galactic cosmic rays: I-B/C ratio, Astron. Astrophys. 394 (2002) 1039 [astro-ph/0206286] [SPIRES]. 
[51] G. Di Bernardo, C. Evoli, D. Gaggero, D. Grasso and L. Maccione, Unified interpretation of cosmic-ray nuclei and antiproton recent measurements, arXiv:0909.4548 [SPIRES].

[52] T.K. Gaisser and R.K. Schaefer, Cosmic-ray secondary antiprotons - a closer look, Astrophys. J. 394 (1992) 174.

[53] F.W. Stecker, M.A. Malkan and S.T. Scully, Intergalactic photon spectra from the far IR to the $U V$ Lyman limit for $0<z<6$ and the optical depth of the universe to high energy gamma-rays, Astrophys. J. 648 (2006) 774 [astro-ph/0510449] [SPIRES]; F.W. Stecker, M.A. Malkan and S.T. Scully, Corrected table for the parametric coefficients for the optical depth of the universe to gamma-rays at various redshifts, Astrophys. J. 658 (2007) 1392 [astro-ph/0612048] [SPIRES].

[54] O. Adriani et al., A new measurement of the antiproton-to-proton flux ratio up to $100 \mathrm{GeV}$ in the cosmic radiation, Phys. Rev. Lett. 102 (2009) 051101 [arXiv:0810.4994] [SPIRES].

[55] AMS-02 collaboration, S. Di Falco, Indirect dark matter search with AMS-02, astro-ph/0607100 [SPIRES].

[56] A.V. Kravtsov, A.A. Klypin, J.S. Bullock and J.R. Primack, The cores of dark matter dominated galaxies: theory vs. observations, Astrophys. J. 502 (1998) 48 [astro-ph/9708176] [SPIRES].

[57] L. Bergstrom, P. Ullio and J.H. Buckley, Observability of gamma rays from dark matter neutralino annihilations in the Milky Way halo, Astropart. Phys. 9 (1998) 137 [astro-ph/9712318] [SPIRES].

[58] A.W. Strong, I.V. Moskalenko and O. Reimer, Diffuse continuum gamma rays from the galaxy, Astrophys. J. 537 (2000) 763 [Erratum ibid. 541 (2000) 1109] [astro-ph/9811296] [SPIRES].

[59] F. Melia, An accreting black hole model for Sagittarius A, Astrophys. J. 387 (1992) L25.

[60] C. Heiles, The local direction and curvature of the galactic magnetic field derived from starlight polarization, Astrophys. J. 462 (1996) 316.

[61] G. Dobler, D.P. Finkbeiner, I. Cholis, T.R. Slatyer and N. Weiner, The Fermi haze: a gamma-ray counterpart to the microwave haze, arXiv:0910.4583 [SPIRES].

[62] M. Ackermann, Extragalactic diffuse, Talk at FERMI Symposium, U.S.A. (2009).

[63] M. Papucci and A. Strumia, Robust implications on dark matter from the first Fermi sky gamma map, JCAP 03 (2010) 014 [arXiv:0912.0742] [SPIRES].

[64] L. Bergstrom, T. Bringmann and J. Edsjo, New positron spectral features from supersymmetric dark matter — a way to explain the PAMELA data?, Phys. Rev. D 78 (2008) 103520 [arXiv:0808.3725] [SPIRES].

[65] L. Bergstrom, T. Bringmann, M. Eriksson and M. Gustafsson, Gamma rays from Kaluza-Klein dark matter, Phys. Rev. Lett. 94 (2005) 131301 [astro-ph/0410359] [SPIRES].

[66] M. Cirelli, P. Panci and P.D. Serpico, Diffuse gamma ray constraints on annihilating or decaying dark matter after Fermi, arXiv:0912.0663 [SPIRES].

[67] T. Sjöstrand et al., High-energy physics event generation with PYTHIA 6.1, Comput. Phys. Commun. 135 (2001) 238 [hep-ph/0010017] [SPIRES]. 